\title{
О ВЛИЯНИИ ФОРМЫ ИМПУЛЬСА НА ПЕРСПЕКТИВЫ КАРОТАЖНОГО РАДИОЗОНДИРОВАНИЯ
}

Волкомирская Л. Б., Гулевич О. А., Резников А. Е.

\begin{abstract}
Аннотация
Успехи метода георадиолокации при исследованиях сред с поверхности подталкивают к идее создания георадара для каротажного радиозондирования. Однако особенности условий работы приборов на большой глубине, в скважине, в окружении среды с высоким поглощением электромагнитных волн, предъявляют дополнительные требования к оптимизации технологии георадиолокации для целей каротажа.

В работе обсуждаются два возможных подхода к каротажному радиозондированию на примере принятых за возможные прототипы георадаров. Анализируются экспериментальные данные, полученные в ходе международной экспедиции в одних и тех же условиях с помощью георадаров MALA (Швеция) и ГРОT 12 (Россия) построенных по разным схемам. Делаются выводы о перспективности использования видеоимпульсов в каротажном радиозондировании.
\end{abstract}

\section{Ключевые слова:}

георадар, каротажный георадар, георадиолокация, georadar, gpr logging, gpr 


\title{
О ВЛИЯНИИ ФОРМЫ ИМПУЛЬСА НА ПЕРСПЕКТИВЫ КАРОТАЖНОГО РАДИОЗОНДИРОВАНИЯ
}

\author{
Л.Б. Волкомирская ${ }^{1,2}$, О.А. Гулевич ${ }^{1,2}$, А.Е. Резников ${ }^{1}$ \\ ${ }^{1}$ Институт земного магнетизма, ионосферы и распространения радиоволн им. Н.В. \\ Пушкова РАН (ИЗМИРАН), г. Москва, г. Троицк \\ ${ }^{2} \mathrm{OOO}$ «Таймер», г. Москва, г. Троицк
}

\begin{abstract}
ВВЕДЕНИЕ
Максимальная глубина исследования околоскважинного пространства применяемых в настоящее время геофизических методов не превышает нескольких первых метров. Поэтому работа по адаптации технологии георадиолокации, которая в задачах подповерхностного зондирования выходит на глубины до 100 и более метров [Волкомирская и др., 2019], для целей каротажного зондирования представляется актуальной и перспективной.
\end{abstract}

Несмотря на условность разделения имеющих в своей основе электромагнитную природу зондирующих импульсов на радиоимпульсы (в основе которых лежат различные по длительности и способу модуляции квазимонохроматические сигналы) и видеоимпульсы (основой которых служат короткие единичные колебания ударного типа напряжения и тока в излучающих антеннах) их взаимодействие с материальными веществами существенно различается. Отличия во взаимодействии радиоимпульсов и видеоимпульсов с материальными средами находят отражение в теоретическом описании этих процессов. Есть и существенные конструктивные отличия устройств, реализующих эти способы зондирования. Для каждого из них существуют свои ограничения по глубине зондирования и качеству получаемых экспериментальных данных.

В первую очередь экспериментальные, а затем и теоретические результаты последних десятилетий убедительно демонстрируют при исследования природных сред преимущества схем построения аппаратуры для генерации и регистрации не квазимонохроматических, а апериодических сигналов для достижения максимальной глубины зондирования. Причем и теория георадиолокации, и эксперимент развивались, за редким исключением, независимо. Это приводит к проблемам в интерпретации получаемых результатов и даже публичным выступлениям некоторых специалистов об 
экспериментальных данных, полученных при георадиолокационном зондировании видеоимпульсами, которые утверждают, что их «невозможно объяснить». Так происходит потому, что некоторые экспериментальные результаты не находили и не находят качественного и количественного объяснения в рамках традиционных решений уравнений Максвелла в сплошных средах, когда они представляются в виде произведений функций, зависящих либо от координат, либо от времени (разделяющиеся решения), обычно исследуемые с помощью преобразования Фурье. Однако многие из полученных экспериментальных результатов становятся понятнее, если их интерпретировать в рамках аналитических и численных подходов к непериодическим и нестационарным решениям уравнений Максвелла.

В работе акцентируется внимание на естественных преимуществах использования сверхширокополосных видеоимпульсов для зондирования природных сред с сильным поглощением и предлагаются направления развития теории и технологий интерпретации экспериментальных данных. Показана практическая целесообразность использования апериодических импульсов для исследования сред с сильным поглощением, в том числе и для каротажного радиозондирования.

\section{ОСОБЕННОСТИ РАСПРОСТРАНЕНИЯ ВИДЕОИМПУЛЬСОВ В ПРИРОДНЫХ СРЕДАХ}

Для целей данной работы важно сравнение распространения стационарных, квазимонохроматических и нестационарных наносекундных импульсов в стационарных средах с сильной дисперсией для обоснования оптимальной формы сигнала в перспективных системах подповерхностного и каротажного зондирования. Деформация квазимонохроматического импульса в среде с дисперсией традиционно описывается в частотной области методом разложения фазы в ряд по степеням отношения спектральной ширины импульса $(\Delta \omega)$ к несущей частоте $(\omega)$ [Ландау, Лифшиц, 1982]. Однако, такой подход неприменим для видеоимпульсов, для которых это отношение не является малым параметром.

Если отказаться от традиционных допущений о малости и медленности изменений полей, то неразделяющиеся точные аналитические решения позволят создать математическую основу для описания быстропеременных непериодических полей и коротких импульсов в диспергирующих средах. Сама среда при этом предполагается покоящейся и стационарной, а нестационарность пространственно-временной структуры распространяющегося поля связана со значительным изменением его огибающей за 
характерное время, определяемое микроскопическими процессами установления поля в среде.

Физические основы и математический аппарат теории нестационарных волновых процессов рассмотрены на примере задачи о распространении электромагнитного поля в изотропной плазме [Шварцбург, 1998]. Теоретически постановка задачи близка к рассмотренной позже численно в [Волкомирская и др., 2013; Rudenchik et al., 2008]. В этих работах показано, что сверхширокополосный импульс и распространяется, и затухает не так, как радиоимпульс. При этом зафиксировано, что один и тот же электромагнитный импульс будет вести себя по-разному при взаимодействии со средами с разным характерным временем установления в них поля, что определяется строением и составом конкретной среды, характеризующимися электрической индукцией.

Таким образом, в одних случаях зондирующий импульс будет вести себя как «короткий», а в других - как «длинный», в зависимости от величины отношения между характерным временем импульса и характерным временем установления поля в среде, которое определяется микропроцессами взаимодействия зарядов в среде с налагаемым внешним полем. Если ставить вопрос о полном соответствии теоретической модели реально происходящим в среде процессам, то надо решать задачу в кинетическом приближении, что даже в сравнительно простых по составу природных средах пока представляется малореальным из-за многочисленных сложностей.

Вычисление электрической индукции, в частности для синусоидальных волн, выполняется обычно в рамках хорошо развитого и известного приближения геометрической оптики, предполагающего медленные изменения параметров среды и поля. В отличие от этого представление переменных токов в среде с помощью неразделяющихся функций [Шварцбург, 1998] даёт возможность вычислить электрическую индукцию без использования указанного приближения.

\section{МАТЕМАТИЧЕСКАЯ ПОСТАНОВКА ЗАДАЧИ ГЕОРАДИОЛОКАЦИИ ДЛЯ ВИДЕОИМПУЛЬСОВ}

Электромагнитное поле зондирующего сигнала в среде задается уравнениями Максвелла:

$$
\operatorname{div} D=0 \quad, \quad \operatorname{div} B=0
$$




$$
\operatorname{rot} E=-\frac{1}{c} \frac{\partial B}{\partial t} \quad, \quad \operatorname{rot} H=\frac{1}{c} \frac{\partial D}{\partial t}+\frac{4 \pi}{c} J
$$

Индукция D связана с электрическим полем $\mathbf{E}$ и током $\mathbf{j}$ через принцип причинности - уравнение (3). Из него следует связь между действительной и мнимой частями диэлектрической проницаемости, называемая формулами Крамерса - Кронинга [Ландау, Лифшиц, 1982].

$$
\begin{aligned}
& D(t)=E(t)+\int_{0}^{\infty} f(\tau) E(t-\tau) d \tau \\
& B=H
\end{aligned}
$$

где $\mathrm{f}(\tau)$ функция, зависящая от параметров J, задающих зондирующий сигнал и от пространственных и от временных параметров среды. В моделях сигналов с ограниченным спектром т.е. квазисинусоидальных радиоимпульсов, эта связь зачастую нарушается, за исключением случая, когда действительная часть диэлектрической проницаемости - произвольная постоянная, а мнимая часть обусловлена только проводимостью, что в уравнении (3) соответствует $f(\tau, x)=C_{1}(x) \delta(\tau)+C_{2}(x) \theta(\tau)$, где $\delta(\tau)$ - функция Кронекера, $\theta(\tau)$ - функция Хевисайда. Для того чтобы не нарушался принцип причинности, диэлектрическую проницаемость можно параметризовать коэффициентами действительной функции $f(\tau, x)$ [Волкомирская и др., 2013].

Электрическая индукция D, определенная формулой (3), связана с электрическим полем Е и током J, наведенным этим полем определяется, как известно, динамическими процессами установления поля в среде. Вклад таких процессов описывается интегральным членом в (3), вычисление которого для ряда полей и, в частности, для синусоидальных волн выполняется обычно в рамках приближения медленных изменений параметров среды и поля. Для быстро меняющихся электромагнитных полей представление переменных токов в среде с помощью неразделяющихся функций дает возможность вычислить интегральный член в (3) в явной форме и это приводит к сложной зависимости скорости потока энергии от координат и времени. А поток энергии при импульсном возбуждении среды зависит ещё и от формы, и от длительности возбуждающего видеоимпульса [Шварцбург, 1998]. 
Видеоимпульсы существенно отличаются по модельному представлению от традиционно обсуждаемых модулированных квазимонохроматических сигналов с прямоугольной или гауссовой огибающей:

1. Огибающая видеоимпульса содержит одно или несколько колебаний поля, формы которых далеки от синусоидальных и по амплитуде, и по расстоянию между точками пересечения оси нулевого потенциала.

2. Передний и задний фронты импульса несимметричны.

Традиционные модели $\delta$-функции или ступенчатой функции Хэвисайда, соответствующие нулевой длительности сигнала и нулевому времени установления, не годятся для рассматриваемой задачи. Применение более реалистических моделей, например, модулированных гауссовых или прямоугольных импульсов ограничено предположением о равенстве интервалов между точками пересечения нуля

$$
f_{1}=\exp \left(\frac{-t^{2}}{2 t_{0}^{2}}\right) \sin \omega t, f_{2}=\left\{\begin{array}{c}
\sin \omega t,|T| \leq \frac{T}{2} \\
0,|T|>\frac{T}{2}
\end{array},\right.
$$

В отличие от этого, в [Шварцбург, 1998] предложено гибкое моделирование реальных видеоимпульсов, содержащих одно или несколько колебаний поля на основе использования функций Лаггера ( $\left.L_{m}\right)$.

$$
L_{m}(x)=\frac{\exp (x / 2)}{m !} \frac{d^{m}}{d x^{m}}\left[\exp (-x) x^{m}\right], x=\frac{t-z c^{-1}}{t_{0}}
$$

где $\mathrm{t}_{0}$ - временной масштаб сигнала.

Сочетание неразделяющихся решений уравнений Максвелла для полей внутри диспергирующих сред и лагерровых представлений импульсных полей вне этих сред образует точно решаемую модель распространения видеоимпульсов в однородной среде и одномерном приближении.

Коэффициенты отражения сигналов (6) находятся, как обычно, из условий непрерывности электрических и магнитных составляющих полей на границе среды ( $z=0 \quad)$

$$
R=\frac{-2 \beta}{1+2 \beta+\sqrt{1+4 \beta}}
$$


где $\beta=\frac{t_{0}}{T}, \quad t_{0}-$ временной масштаб сигнала, $\mathrm{T}-$ время становления поля в среде.

$$
\mathrm{T}=\varepsilon_{\infty} / 2 \pi \sigma
$$

Зависимость коэффициента отражения от отношения характерных времен показана на рис. 1. Как видно, отражение импульсов усиливается с ростом отношения $\beta=\frac{t_{0}}{T}$, а это значит, что одни и те же границы сред с разными электрическими свойствами становятся более контрастными для более коротких импульсов.

Полученные качественные результаты можно использовать как для понимания, так и интерпретации экспериментальных данных, систематически получаемых в многочисленных экспериментах последних десятилетий. Так, полученные результаты точного расчета показывают, что непериодические сигналы могут затухать в проводящей среде медленнее, чем синусоидальные волны. При этом распространение сигнала в проводящих средах сопровождается искажением его формы [Волкомирская и др., 2013]. Существенно, что нестационарные поля в проводящей среде характеризуются естественным масштабом времени Т (7). Определяющая роль временных параметров в процессах импульсного возбуждения таких полей в сплошных средах показана в [Шварцбург, 1998, Rudenchik et al., 2008].

\section{СРАВНЕНИЕ ЭКСПЕРИМЕНТАЛЬНЫХ ДАННЫХ ГЕОРАДАРОВ РАМАК И ГРОТ $12 \mathrm{H}$}

Рассмотрим экспериментальные данные, полученные при прохождении одних и тех же профилей приборами разной конструкции, для анализа зависимости распространения импульсных сигналов разной формы на глубину и разрешающую способность радиозондирования. Описываемая работа выполнялась в рамках проекта «Быковский» весной 2017 г. в Тикси (Якутия) по договору с Институтом им. Альфреда Вегенера - Центр полярных и морских исследований им. Гельмгольца (АВИ).

Совместные исследования проводились на пресном озере Гольцовое группой российских исследователей с георадаром ГРОТ 12Н, производства ООО «Таймер» (рис. 2), и немецких исследователей с георадаром PAMAK шведского производства компании Mala GeoScience с антеннами для пересеченной местности RTA 50 МГц (II) (рис.3). Значения 50 МГц (РАМАК) и 75 МГц (ГРОТ 12Н) определяют наиболее эффективно излучаемую и принимаемую частоту в спектре каждого из приборов. 
Известно, что приемная часть георадара РАМАК, одного из передовых производителей георадаров использующих радиоимпульсы, построена на принципе частотного стробирования [Финкельштейн и др., 1994], когда для восстановления волновой формы, принятый сигнал переносится в низкочастотную область, в которой и происходит аналого-цифровое преобразование. Для отображения отражённого сигнала на временной шкале, например, из 512 точек с шагом 1 нс, требуется 512 импульсов передатчика. Поэтому одной из существенных характеристик георадаров, построенных на принципах стробирования, является скорость следования импульсов передатчика. Так, для георадаров серии РАМАК эффективная частота повторения зондирующих импульсов достигает 100 кГц на физический канал (https://www.guidelinegeo.com/wpcontent/uploads/2016/03/MALA-RTA-Antennas.pdf). Частотное стробирование приводит, кроме внесения дополнительных помех в тракт приёма, к полосовой фильтрации отраженного сигнала, с потерей низкочастотных составляющих и, тем самым, к уменьшению предельно достижимой глубины зондирования.

Действительно, стробоскопический преобразователь при дискретизации сигнала во времени берет один отсчет от каждой копии сигнала (в отличие от АЦП). Это позволяет преобразовать сигнал в последовательность импульсов, огибающая которых повторяет форму сигнала, а длительность может быть значительно больше исходной. Период стробирующего импульса $T_{c u}$, как правило, превышает период сигнала $T_{c}$ на шаг считывания $\Delta t: T_{c u}=m * T_{c}+\Delta t$. При коэффициенте $m>1$ пропущен ряд периодов сигнала, обычно $m=1$. Для получения формы всего сигнала на периоде $T_{c}$ требуется минимум n периодов стробимпульсов $n=T_{c} / \Delta t \quad$ (каждый из стробимпульсов смещен на шаг считывания). Тогда общая длительность преобразованного сигнала (последовательности $\mathrm{n}$ стробимпульсов, промодулированных по амплитуде входным сигналом) равна $T_{n p}=n * T_{c u} \quad$ и коэффициент временного преобразования (растяжения масштаба по времени) составляет

$$
K_{n p}=\frac{T_{n p}}{T_{c}}=\frac{n \cdot T_{c u}}{T_{c}}=\frac{T_{c u}}{\Delta t} \approx n
$$

Увеличение $K_{n p}$ ограничено техническими возможностями преобразователя:

• минимальным шагом считывания;

• быстродействием;

- стабильностью шага считывания;

• длительностью стробимпульса. 
Расширение сигнала во временной области эквивалентно сужению его в частотном представлении спектра. Поэтому применяют понятие эффективной полосы пропускания, которая зависит от максимально достижимой частоты следования стробирующих импульсов. Обычно ее связывают с минимальной длительностью стробимпульса $\tau_{c u}$, эмпирическим соотношением

$$
f_{B}=(0.45-0.6) / \tau_{c u}
$$

Для рассматриваемого аппаратного комплекса РАМАК оцифровка отраженного сигнала осуществляется через $\Delta \mathrm{t}=2 \mathrm{Hc}$, а скорость получения волновой формы отраженного сигнала позволяет регистрировать 2-4 волновые формы в секунду, в зависимости от настройки прибора.

В моноимпульсном георадаре ГРОТ 12Н происходит прямая оцифровка отраженного сигнала от одного мощного (не менее 1 МВт) видеоимпульса на всю глубину предполагаемых временных задержек - до 20000 нс (http://www.georadargrot.com/grot12h). Отсутствие стробирования позволяет проводить оцифровку в этом диапазоне без сужения спектра. Погрешность оцифровки при этом определяется характеристиками АЦП. Отношение сигнал/шум (Signal to Noise Ratio - SNR), равное отношению среднеквадратического значения величины входного сигнала $V_{i}$ к среднеквадратическому значению величины шума $V_{\text {noise }}$, выраженное в децибелах:

$$
S N R=20 \log \frac{V_{i}}{V_{\text {noise }}} \text {. Величина SNR позволяет определить долю шума в измеряемом }
$$
сигнале по отношению к полезному сигналу. Для АЦП с определенным разрешением именно шум квантования ограничивает возможности преобразователя теоретически лучшим значением отношения сигнал/шум, которое определяется как: $S N R=6,02 N+1,76$, где $\mathrm{N}$ - разрядность АЦП. В частности, для используемого в ГРОТ $12 \mathrm{H}$ 12-разрядного АЦП $S N R=74$ дБ. Шум квантования может быть снижен только путем проведения измерений с помощью АЦП большей разрядности. Увеличение динамического диапазона при заданных характеристиках АЦП осуществляется с помощью линейных аттенюаторов. Количество импульсов передатчика равно количеству волновых форм на радарограмме. Скорость съёмки в пешеходном или транспортном варианте практически не лимитируется скоростью повторения импульсов. Схема прибора позволяет обходиться без процедуры накопления и усреднения, хотя и допускает такую возможность.

Основным элементом при формировании видеоимпульсного сигнала являются передающая и приемная антенны. Для георадаров любых конструкций они, кроме прочего, 
выполняют функции фильтров, как для приёмного, так и для передающего сигнала. Существенным отличием конструкции ГРОТ 12Н и РАМАК является реализация формирования сигналов разной формы. Конструкция георадаров серии ГРОТ 12 с помощью резистивной нагрузки позволяет получать апериодический зондирующий импульс, форма которого обеспечивает большую крутизну фронта и отсутствие дополнительных осцилляций, связанных с переотражениями излучаемого и принимаемого сигнала от концов антенн. Излучающая антенна ГРОТ 12 рассеивает почти половину мощности сигнала передатчика на нагрузочных сопротивлениях, но за счёт большой мощности единичного сверхширокополосного апериодического импульса, который легко сформировать газоразрядным или твёрдотельным ключевым элементом, получается значительный выигрыш в эффективном динамическом диапазоне всего устройства. На практике, напряжение ударного возбуждения в георадаре РАМАК составляет 370 В, а в георадаре ГРОТ 12 не менее 5 кB. Технические характеристики РАMAK RTA50МГц и ГРОТ 12Н приведены на сайтах разработчиков (https://www.guidelinegeo.com/ и http://www.georadargrot.com/grot12h).

Для сопоставления данных зондирования георадарами РАМАК и ГРОТ 12Н выбран профиль, на котором последовательно выполнены исследования с двумя разными георадарами и антеннами.

На рисунке 4 показано взаимное расположение профилей на поверхности озера Гольцовое в окне программы GROT, красной линией выделен профиль, пройденный с севера на юг по льду озера георадаром ГРОТ 12Н с антеннами длиной 2м (что соответствует эффективной центральной частоте излучения 75 МГц). Для удобства совместного анализа на рис. 5 и рис. 6 выведены радарограммы с обоих георадаров, полученные на одном и том же профиле.

Мы провели анализ радарограмм в независимой программе обработки данных SeiSee, разработанной изначально для обработки сейсмических данных, но вполне применимой к обработке радарограмм, полученных в георадиолокации. При этом были применены одинаковые ослабления и фильтрации.

Радарограммы с обоих георадаров, полученные в описываемом эксперименте на одном и том же профиле в одинаковых условиях, представляют измеренное на поверхности льда поле отраженного сигнала.

Внешний вид радарограммы РАМАК (рис. 6) носит более сглаженный характер по сравнению с радарограммой ГРОТ $12 \mathrm{H}$ (рис. 5), но на них отражены одни и те же геологические структуры. Шкала временных задержек РАМАК ограничена количеством 
точек в соответствии с техническими характеристиками. Сравнение рис. 5 и 6 показывает, что для корректного сопоставления данных требуется совмещение радарограмм и по профилю и по оси временных задержек. Для сопоставления положения границ отраженного сигнала и, соответственно, оценки относительной глубины зондирования необходимо сдвинуть радарограмму ГРОТ 12Н вниз по оси временных задержек на 50 нс.

\section{СРАВНЕНИЕ ЭКСПЕРИМЕНТАЛЬНЫХ ДАННЫХ НА ОСНОВЕ АНАЛИЗА СПЕКТРОВ ОТРАЖЕННОГО СИГНАЛА}

В нашем эксперименте данные от двух разных георадаров получены на одном и том же профиле в одно и то же время, поэтому параметры среды можно считать одинаковыми. Попробуем проанализировать с учетом этого полученные данные для оценки особенностей зондирующих импульсов в обоих случаях.

Сравним спектры отраженных сигналов в одних и тех же точках на профиле и применим процедуру усреднения по всему профилю. На всех рисунках, на которых ниже представлены спектры, по горизонтальной оси отложена частота, а по вертикальной относительная амплитуда отражённого сигнала.

На рисунке 7 представлена амплитудно-частотная характеристика (АЧХ) для двух георадаров при одинаковом значении величины временных задержек. По вертикальной оси отложено относительное значение амплитуд гармоник отраженного сигнала в процентах их присутствия усредненное по всему профилю, по горизонтальной частота в МГц. В целом, спектр сигнала для РАМАК (пунктиром на рис. 7) соответствует проведенным выше оценкам, имеет более сглаженный (усреднённый) характер. Верхняя граница ограничена в два раза меньшей частотой из-за меньшей в два раза частоты оцифровки. Граница спектра в области низких частот у РАМАК, как и следовало ожидать, приближена к центральной частоте антенны - 50МГц. Особенности спектра сигнала РАМАК является следствием использования в схеме приёмника частотного стробирования. Действительно, схема стробирования [Финкельштейн и др., 1994] приводит к тому, что все три параметра (скорость следования импульса, количество точек оцифровки на шкале временных задержек и длина шкалы) жёстко связаны.

Разрешающая способность по глубине определяется высокочастотной частью спектра импульса. Как было показано, например, в [Финкельштейн и др., 1994], разрешающая способность по глубине определяется длительностью импульсов $\tau_{0,5} \quad$ на уровне 0,5 от максимальной амплитуды: чем меньше $\tau_{0,5}$, то есть, чем быстрее спадает 
амплитуда до половины от своего максимума, тем выше разрешающая способность. В то же время глубина зондирования определяется низкочастотной частью спектра импульса. Таким образом, ширина спектра определяет и разрешающую способность и глубинность георадара, то есть его реальный динамический диапазон, подтверждаемый на практике.

Проанализируем эффективную ширину спектра отраженного сигнала для используемых георадаров на основе полученных данных (рис.7). Ширина спектра принимаемого сигнала на уровне $A=40 \%$ от максимальной амплитуды составляет для ГРОТ 12Н около 100 МГц, а на уровне $A=20 \%$ около 225 МГц. У георадара РАМАК эти величины составляют 30 МГц и 90 МГц соответственно.

В высокочастотной части спектра у ГРОТ 12Н амплитуда отражённого сигнала в пределе сходятся к значению $A=10 \%$, а у георадара РАМАК - к $A=0 \%$. В [Харкевич, 2009] показано, что скорость убывания амплитуд гармоник в спектре зависит от структурных свойств сигнала: коэффициенты убывают тем быстрее, чем более «гладкой» является форма сигнала и его производных. Если сигнал имеет скачкообразные переходы, что характерно для одиночного видеоимпульсного сигнала, амплитуды гармоник в его спектре стремятся к нулю очень медленно. Чем быстрее убывают коэффициенты Фурье, чем более «гладкая» форма сигнала, тем меньше ширина его спектра. В пределе имеет место наиболее «гладкое» моногармоническое колебание.

Таким образом, зафиксированные отличия в спектрах отражают особенности формы сигнала в рассматриваемых георадарах и свидетельствуют, что в георадаре ГРОТ 12 сигнал видеоимпульсный, в отличие от радиоимпульсного в георадаре РАМАК.

Радарограмма профиля, пройденного георадаром ГРОТ 12Н до глубин временных задержек 800 нс (рис.8), позволяет продолжить анализ отраженного сигнала и на больших глубинах.

\section{ОБСУЖДЕНИЕ РЕЗУЛЬТАТОВ}

Сравнение радарограмм на рис. 5 и 8 показывает, что визуализация радарограммы с увеличенной в 2 раза шкалой временных задержек, полученной с ГРОТ $12 \mathrm{H}$ позволила зафиксировать зоны различного характера переотражений до временных задержек свыше 700 нс, а для георадара РАМАК до 400 нс.

На радарограммах (рис. 5, 6) заметны различия коэффициентов отражения от неоднородностей, например, в районе интервала временных задержек 350-400 нс, что выражается в степени контрастности отображения подповерхностных структур. В целом, 
наблюдается большее затухание сигнала для георадара РАМАК. Следует учесть, что центральная частота у георадара РАМАК сдвинута в низкочастотную часть спектра на 25 МГц, по отношению к центральной частоте Грот 12Н. Если бы все характеристики у приборов были одинаковы, это должно было бы привести к зондированию на заметно большую (примерно в 1,5 раза) глубину РАМАК (согласно классическим представлениям теории геометрической оптики).

Для практического применения в каротажном радиозондировании отметим различия в габаритах антенных систем. При последовательном расположении приемной и передающей антенны в ГРОТ 12Н, как это сделано в наземном исполнении для РАМАК RTA 50МГц, общая длина установки составит 5 метров, у PAMAK RTA 50 МГц общая длина 9,25 метра. Разница для георадара в каротажном исполнении весьма существенная. Следует отметить, что для обеспечения радиального разрешения каротажный радар должен содержать еще, как минимум, одну приемную антенну [Гулевич и др., 2014], что приведет к еще большей общей длине.

Сравнение спектров на рис. 7 позволяет выявить следующие особенности:

1) наличие заметной низкочастотной составляющей в спектре ГРОТ $12 \mathrm{H}$ и ее отсутствие в спектре РАМАК;

2) заметное увеличение в обе стороны эффективной полосы частот принимаемого сигнала у ГРОТ 12H;

3) существенно большее присутствие высокочастотных составляющих в спектре ГРОТ $12 \mathrm{H}$.

Отмеченные различия в рассматриваемых спектрах отражают тот факт, что форма зондирующего сигнала у георадара РАМАК представляется радиоимпульсом [Харкевич, 2009], а у георадара ГРОТ 12Н соответствует видеоимпульсу. Об этом свидетельствует и значительное присутствие низкочастотных составляющих в спектре отражённого сигнала видеоимпульса и медленное стремление к нулю амплитуд гармоник высокочастотных составляющих.

При увеличении шкалы временных задержек для расчета спектра, соответствующего радарограмме на рис.8, амплитудно-частотный спектр (АЧС) однозначно отражает тот факт, что форма сигнала у ГРОТ $12 \mathrm{H}$ близка к форме классического видеоимпульса с крутым передним фронтом [Харкевич, 2009] (рис. 9). К сожалению, удовлетворительной модели взаимодействия таких импульсов с сильнопоглощающими и диспергирующими средами, позволяющей проводить количественные расчёты даже для известных своими свойствами геологических разрезов, пока не создано. 
Можно отметить тот факт, что при зондировании природных сред видеоимпульсами, уже широко применяемыми в разных областях радиолокации и связи, эксперимент пока заметно опережает теорию, позволяя получать уже для достигнутого уровня развития технологии практически значимые результаты для многих приложений.

Рассматриваемые в работе экспериментальные данные опираются на теоретические модели, которые пока представлены в весьма ограниченных приближениях. Для перехода к решению задачи с учетом слоистости дисперсной среды даже в одномерном приближении, необходим переход на численные методы. Численных решений задачи с учетом неразделяющихся уравнений Максвелла при распространении видеоимпульсов внутри диспергирующих сред, в частности, моделирующих геологический разрез скважин, на сегодняшний день не существует. Поэтому для получения количественных оценок преимуществ каротажного зондирования видеоимпульсами приведенное сравнение экспериментальных данных может представлять интерес. В частности, полученные данные об увеличении глубины зондирования в 2 раза при увеличении разрешающей способности, позволяют оценить изменение предельной дальности зондирования в скважине.

Следует отметить, что проблемой при теоретическом моделировании импульсного электромагнитного излучения скважинных георадаров является учёт влияния конструкции излучающей и приёмной антенн, оболочки каротажного зонда и прослойки бурового раствора, присутствующего в скважине, на амплитуду и спектр импульса, излучаемого в зондируемую среду и принимаемого внутри каротажного зонда. Фактически, оболочка каротажного зонда и прослойка бурового раствора должны рассматриваться электродинамически как часть конструкции антенн, так как они находятся в ближней зоне излучателей [Эпов и др., 2008]. Эти особенности должны учитываться и при построении каротажного видеоимпульсного георадара.

\section{ВЫВОДЫ}

Сопоставление данных двух георадаров и теоретические выводы работ [Шварцбург, 1998; Волкомирская и др., 2013; Rudenchik et al., 2008] позволяют объяснить более высокую эффективность приборов, работающих на видеоимпульсах с апериодической формой, по сравнению с радиоимпульсными приборами. Так, в одних и тех же условиях, один и тот же интернациональный коллектив операторов на георадаре ГРОТ 12Н с более высокочастотными антеннами получил отражения с примерно в 2 раза больших глубин, чем на георадаре РАМАК. Отмечено меньшее затухание сигнала в среде в согласии с 
[Шварцбург, 1998], большая разрешающая способность и лучшее отражение от границ сред с различными электрическими характеристиками при использовании видеоимпульсов.

Проведенные исследования показывают, что для получения количественных оценок затухания и разрешающей способности, необходимо адекватно описывать взаимодействие видеоимпульсов со средой и строить решение на основе уравнений Максвелла для быстропеременных непериодических полей. Особенно это актуально в задачах каротажного зондирования и межскважинного радиопросвечивания, где среда характеризуется существенной проводимостью и частотной дисперсией [Эпов и др., 2011] и есть ограничения на массогабаритные характеристики аппаратуры. Исследование конкретных моделей сред нефтегазового коллектора на базе численного моделирования нестационарных неразделяющихся решений уравнений Максвелла поможет оптимизировать конструкцию каротажного георадара и форму видеоимпульса для достижения максимально возможной глубины зондирования с сохранением высокой разрешающей способности. В настоящее время такие расчеты пока не выполнены, поэтому экспериментальные исследования характеристик приборов, построенных на разных принципах, в сопоставлении с точными решениями распространения непериодических сигналов в однородной среде, хотя и являются качественными, но задают, по мнению авторов, вектор перспективы развития технологии каротажного радиозондирования.

Практическая целесообразность проведения экспериментальных работ по использованию видеоимпульсов для каротажного зондирования основана прежде всего на перспективе увеличения максимальной глубинности исследования околоскважинного пространства: до десятков метров при зондировании и до сотен метров при радиопросвечивании (по предварительным оценкам исходя из возможностей прототипа георадара ГРОТ 12Н в подповерхностной георадиолокации).

Представляется весьма полезным в отсутствие оценок на основе численных расчетов задачи распространения видеоимпульсов в среде, характерной геологическим моделям скважинного пространства, провести сравнительный эксперимент по сопоставлению данных видеоимпульсного и радиоимпульсного сигнала в равных условиях для определения количественного увеличения предельной глубины зондирования скважинного пространства при переходе на видеоимпульсы.

\section{БЛАГОДАРНОСТЬ}


Авторы благодарят Институт им. Альфреда Вегенера - Центр полярных и морских исследований им. Гельмгольца (АВИ) за предоставленную возможность совместных исследований. Отдельно выражаем благодарность Michael Angelopoulos за совместную работу, а также благодарим всех участников экспедиции «Bykovsky Spring 2017» за сотрудничество.

\section{СПИСОК ЛИТЕРАТУРЫ}

Волкомирская Л., Гулевич О., Руденчик Е. Георадиолокация в средах с дисперсией. Зависимость амплитуды и формы импульса георадара от дисперсии среды. Из-во LAP (Lambert Academic Publishing), 2013. ISBN 978-3-659-35367-3. 81 с.

Волкомирская Л.Б., Гулевич О.А., Ляхов Г.А., Резников А.Е. Георадиолокация больших глубин. Журнал радиоэлектроники [электронный журнал]. 2019. № 4. Режим доступа: http://jre.cplire.ru/jre/apr19/6/text.pdf. DOI 10.30898/1684-1719.2019.4.6.

Гулевич О.А., Варенков В.В., Волкомирская Л.Б. Ляхов Г.А., Резников, А.Е., Руденчик Е.А., Сахтеров В.И. Способ и система георадиолокационного каротажа. Патент на изобретение № 2550773 по заявке №2014106905/28/(010958) от 25.02.2014г.

Ландау Л.Д., Лившиц Е.М. Электродинамика сплошных сред. М., Наука, 1982, 389 C.

Финкельштейн М.И., Карпухин В.И., Кутев В.А., Метелкин В.Н. Подповерхностная радиолокация. М. Радио и связь, 1994, 216 с.

Харкевич А.А. Спектры и анализ. Либроком, 2009, 240 с.

Шварцбург А.Б. Видеоимпульсы и непериодические волны в диспергирующих средах (точно решаемые модели) // УФН, 1998, Т. 168, № 1, с. 85-103.

Эпов М.И., Савин И.В., Миронов В.Л. Спектроскопические характеристики диэлектрической проницаемости влажных горных пород/ Материалы XII Международной научной конференции «Решетневские чтения». Красноярск, 10-12 ноября 2008. С. 116-117.

Эпов М.И., Миронов В.Л., Музалевский К.В., Сверхширокополосное электромагнитное зондирование нефтегазового коллектора. Новосибирск, Из-во СО РАН, 2011, 114 c.

Rudenchik E.A., Volkomirskaya L.B., and Reznikov A.E. Study of Signal Propagation in One-Dimensional Electrodynamics for Interpretating Electromagnetic Sounding Data. Consideration of Conductivity in the Function of Permittivity // ISSN1541-308X, Physics of Wave Phenomena, 2008, Vol. 16, N 2, p. 1-14. 
Рис. 1. Зависимость коэффициента отражения R видеоимпульса от характерных времен импульса и среды. 
Рис. 2. Георадар ГРОТ 12Н с антенной 2 метра (75МГц). 
Рис. 3. Участник экспедиции Michael Angelopoulos с георадаром РАMAK с антенной 3 метра (50 МГц). 
Рис.4. Схема пройденных георадарных профилей на карте озера Гольцовое в окне программы камеральной обработки GROT. 
Рис. 5. Радарограмма в программе Seisee профиля, выполненного с севера на юг георадаром ГРОТ 12Н с антеннами 2м (центральная частота 75МГц). 
Рис. 6. Радарограмма в программе Seisee того же профиля (как на рис.5), пройденного с георадаром РАMAK RTA 50МГц. 
Рис.7. Спектр отраженного сигнала для РАМАК (а). Спектр отраженного сигнала для ГРОТ 12Н (b). По горизонтали - частота F в МГц, по вертикали - относительная амплитуда гармоник А. 
Рис. 8. Радарограмма профиля ГРОТ 12H в программе SeiSee (соответствующий данной радарограмме спектр сигнала представлен на рис. 9). 
Рис. 9. Спектр отраженного сигнала георадара ГРОТ 12Н на уровне волновых задержек 800 нс. Здесь, как и на рис. 7, по горизонтали - частота F в МГц, по вертикали - относительная амплитуда гармоник А. 


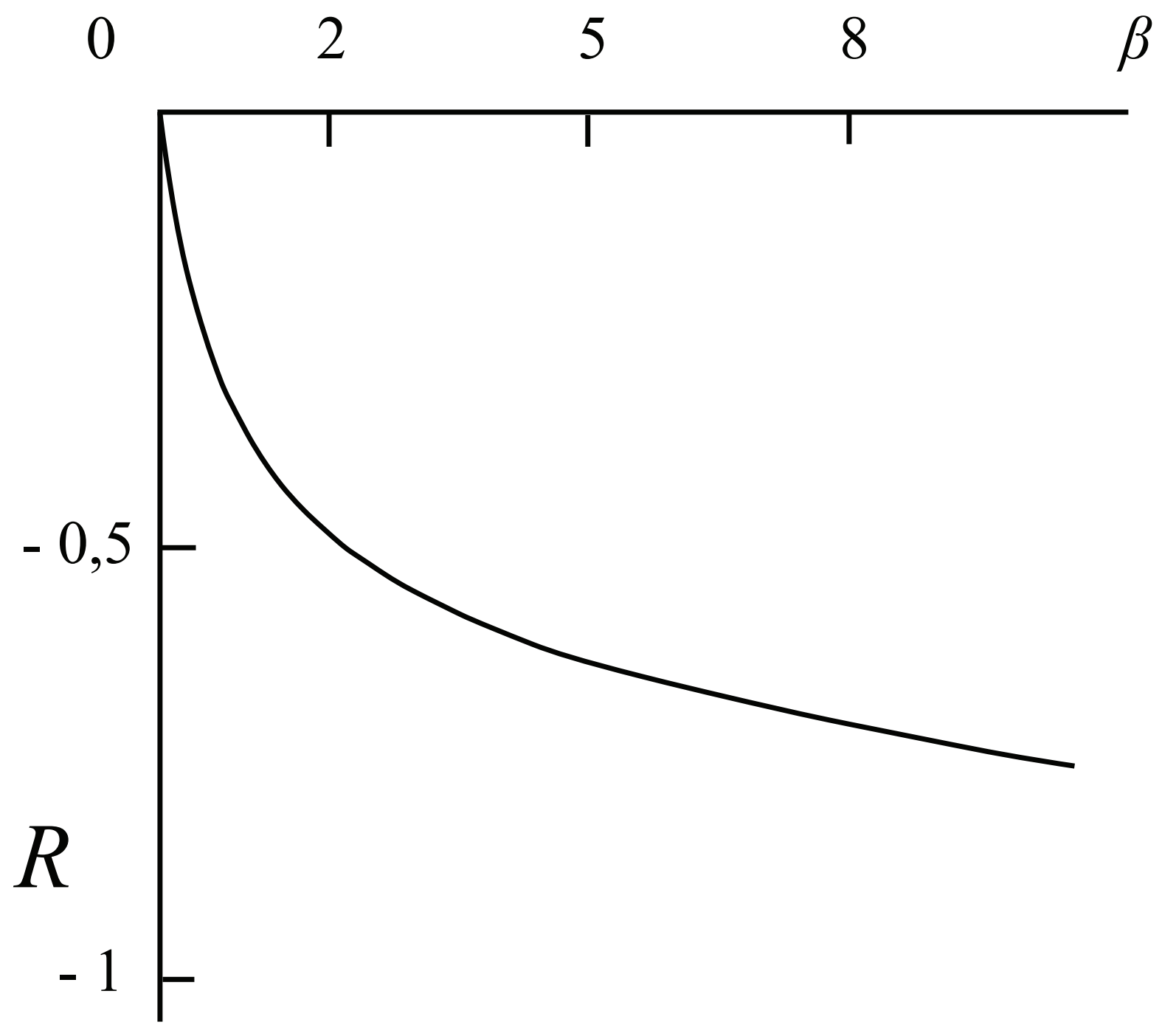




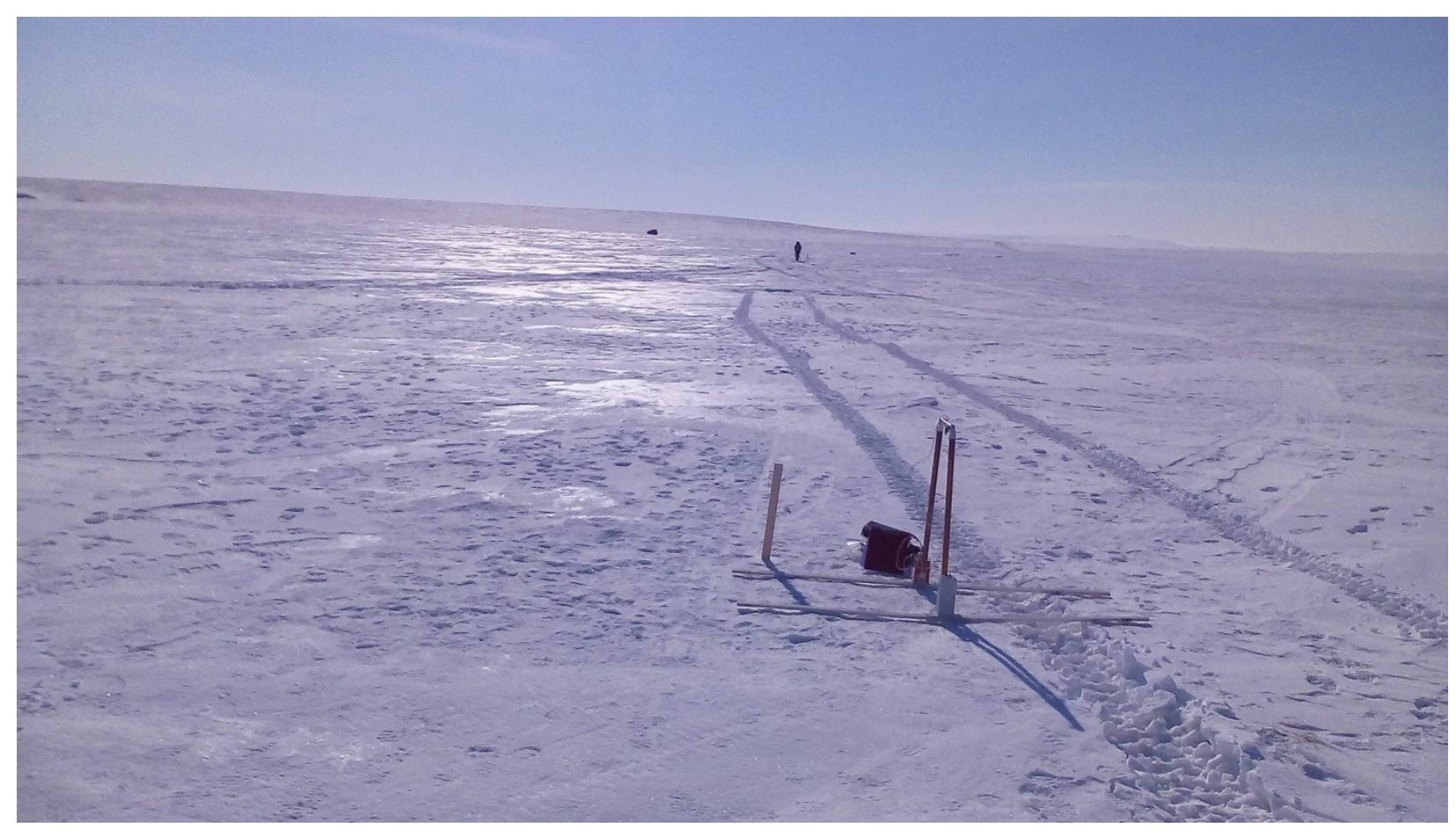




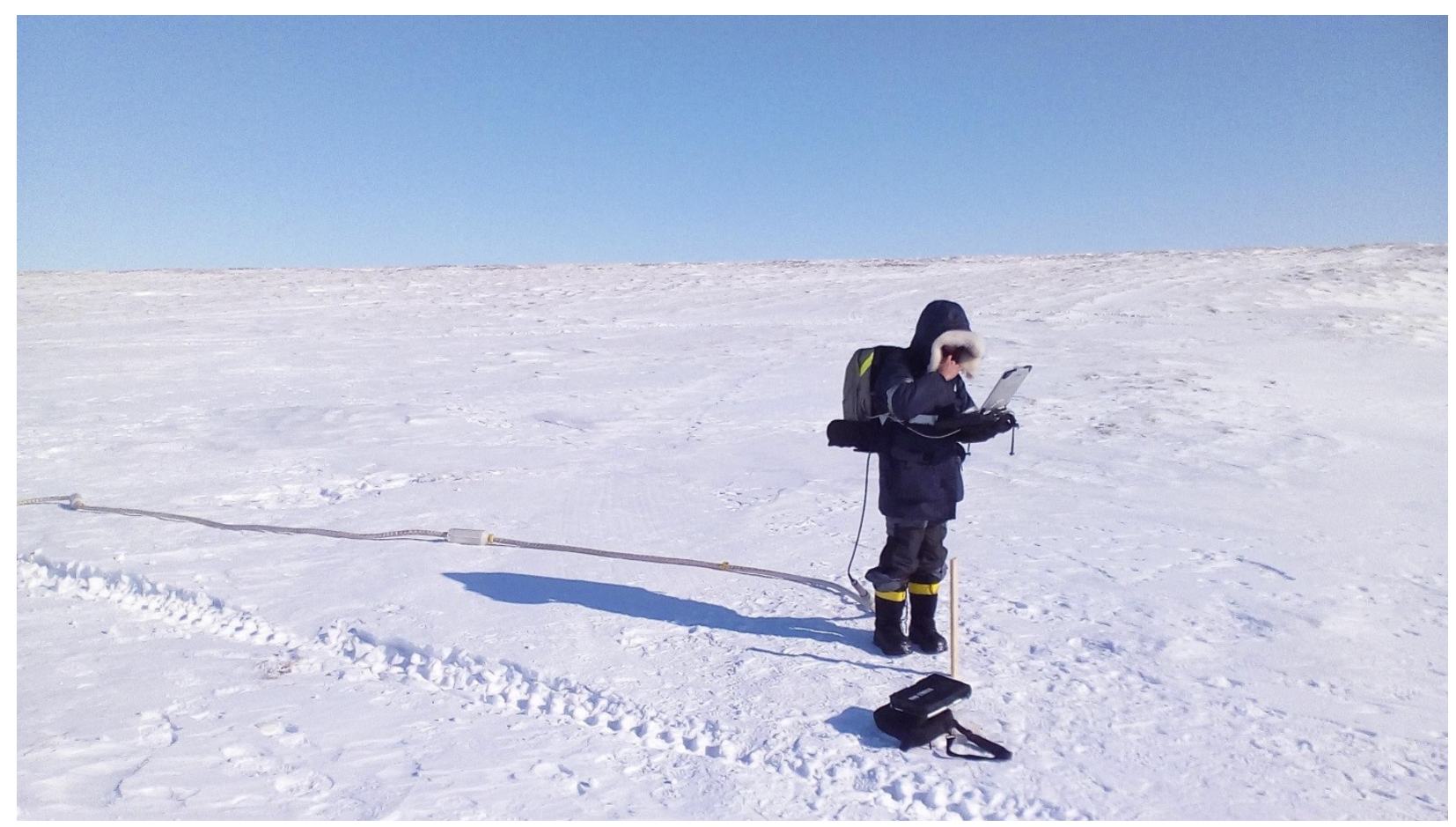




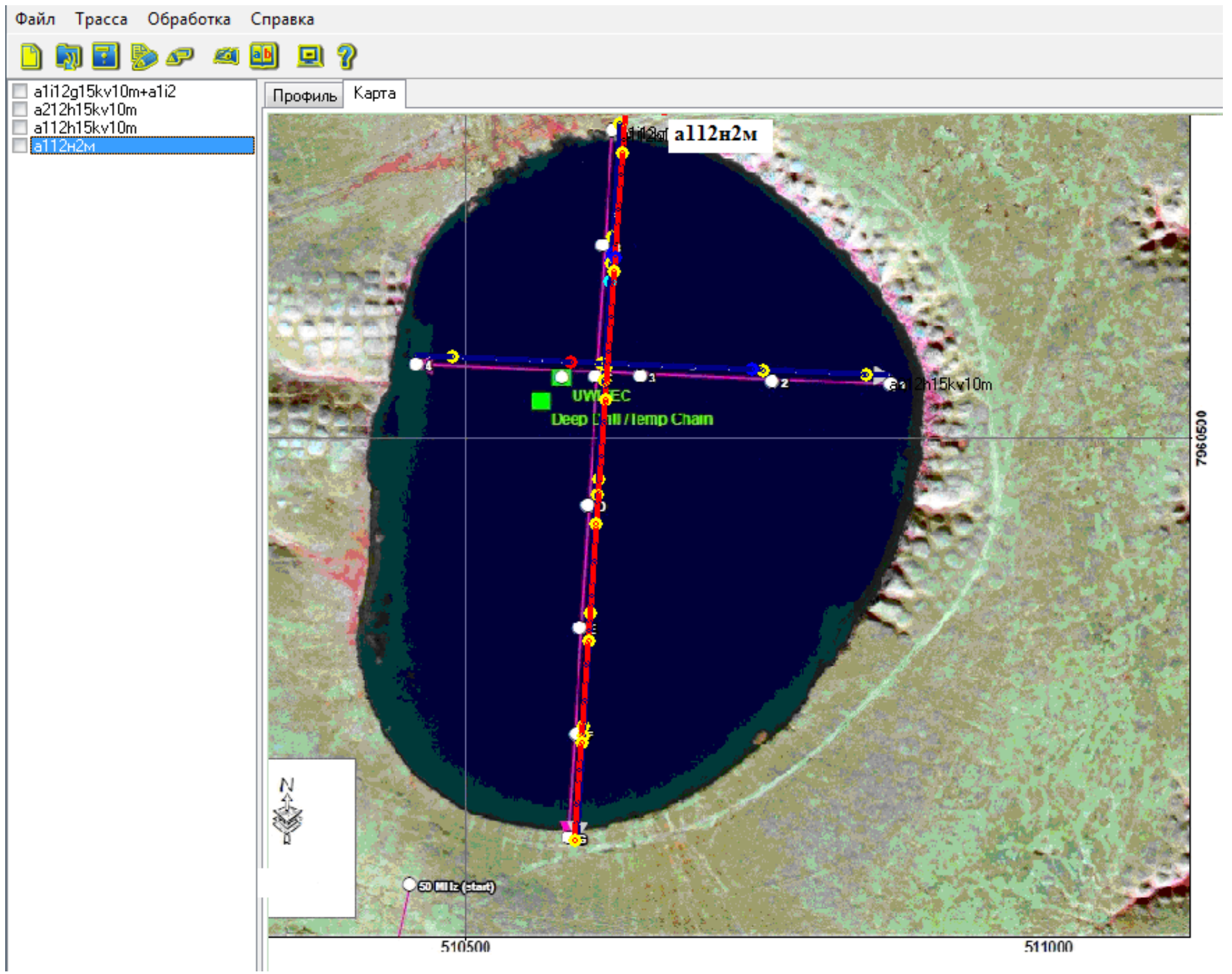




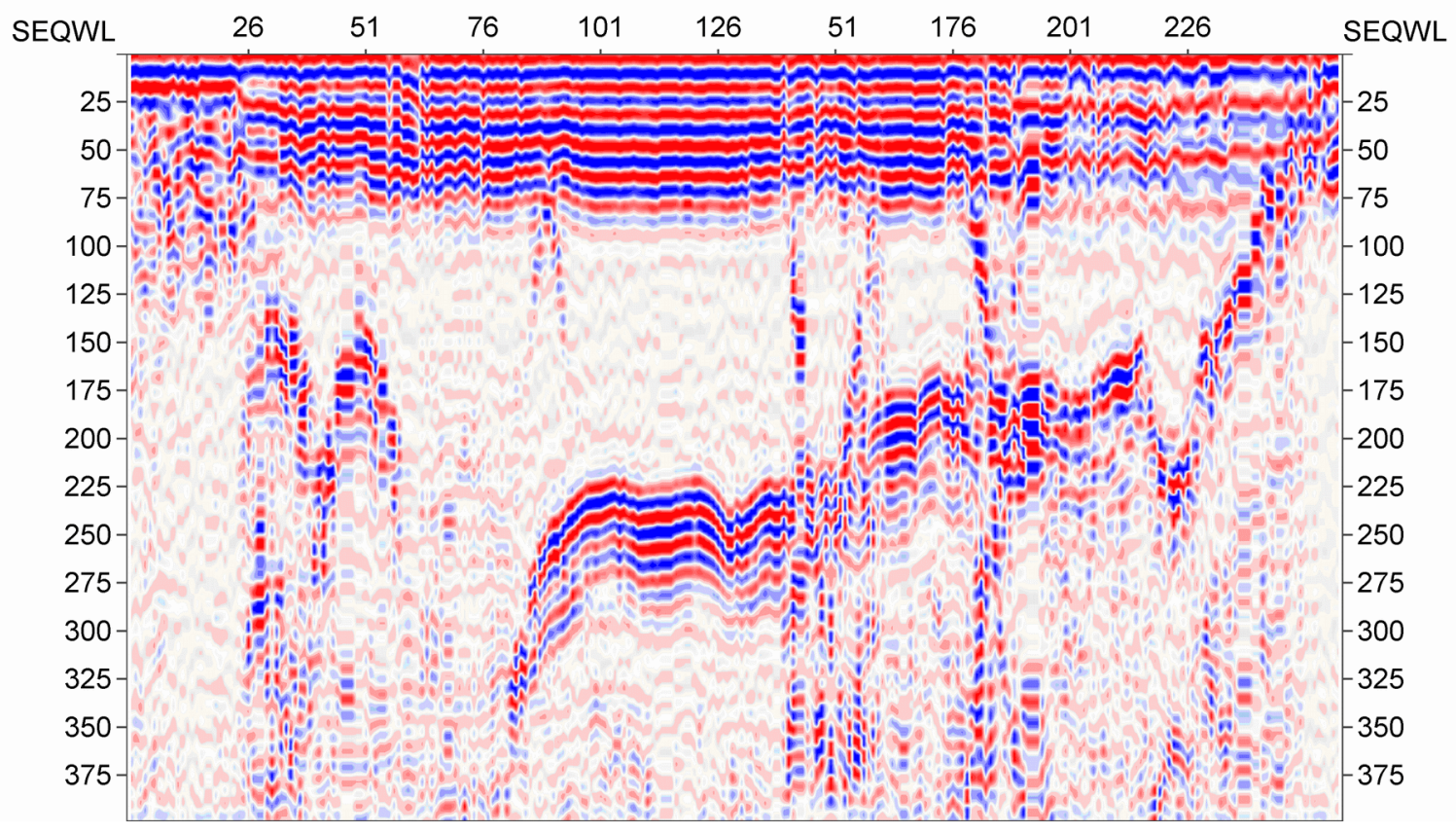




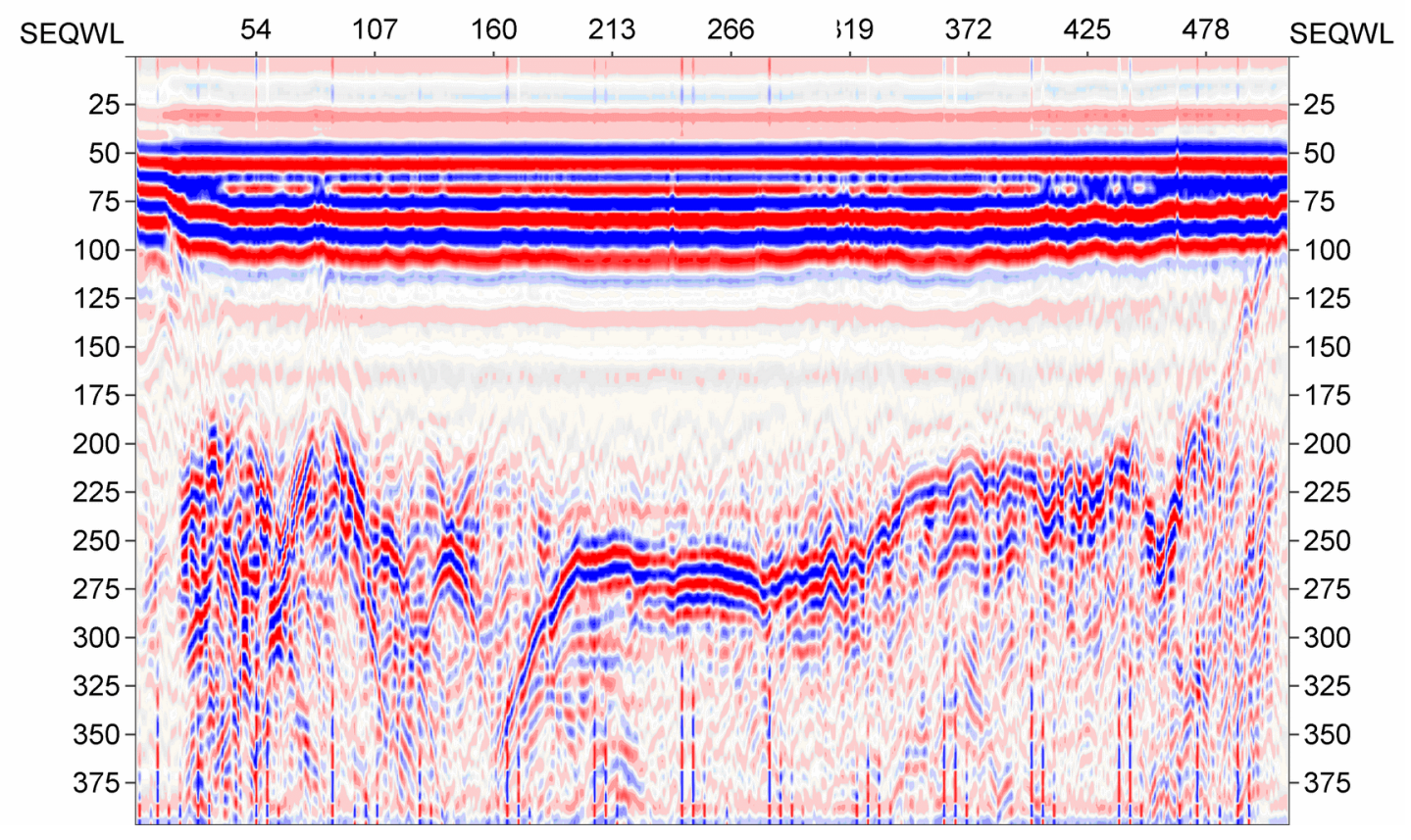



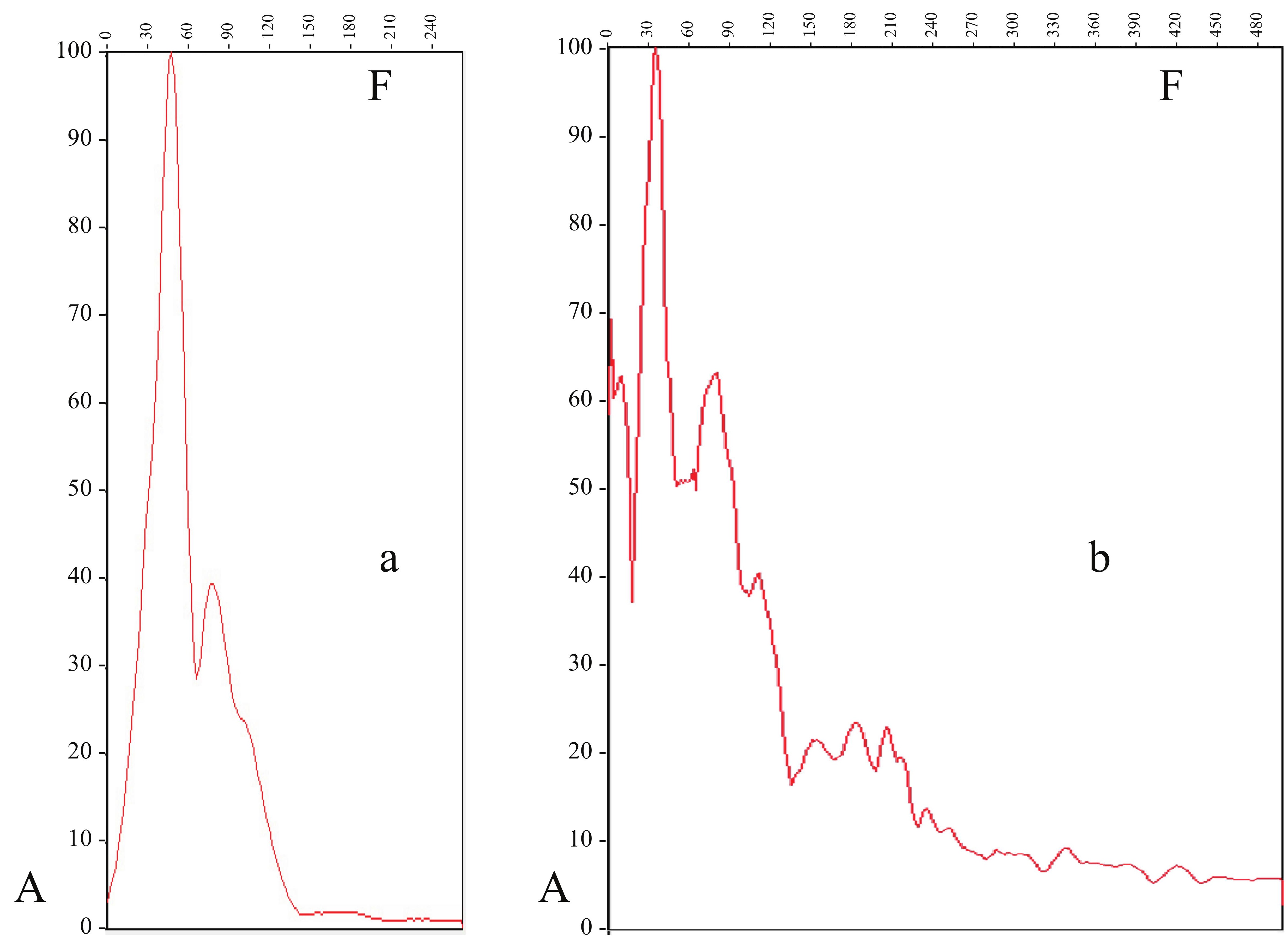


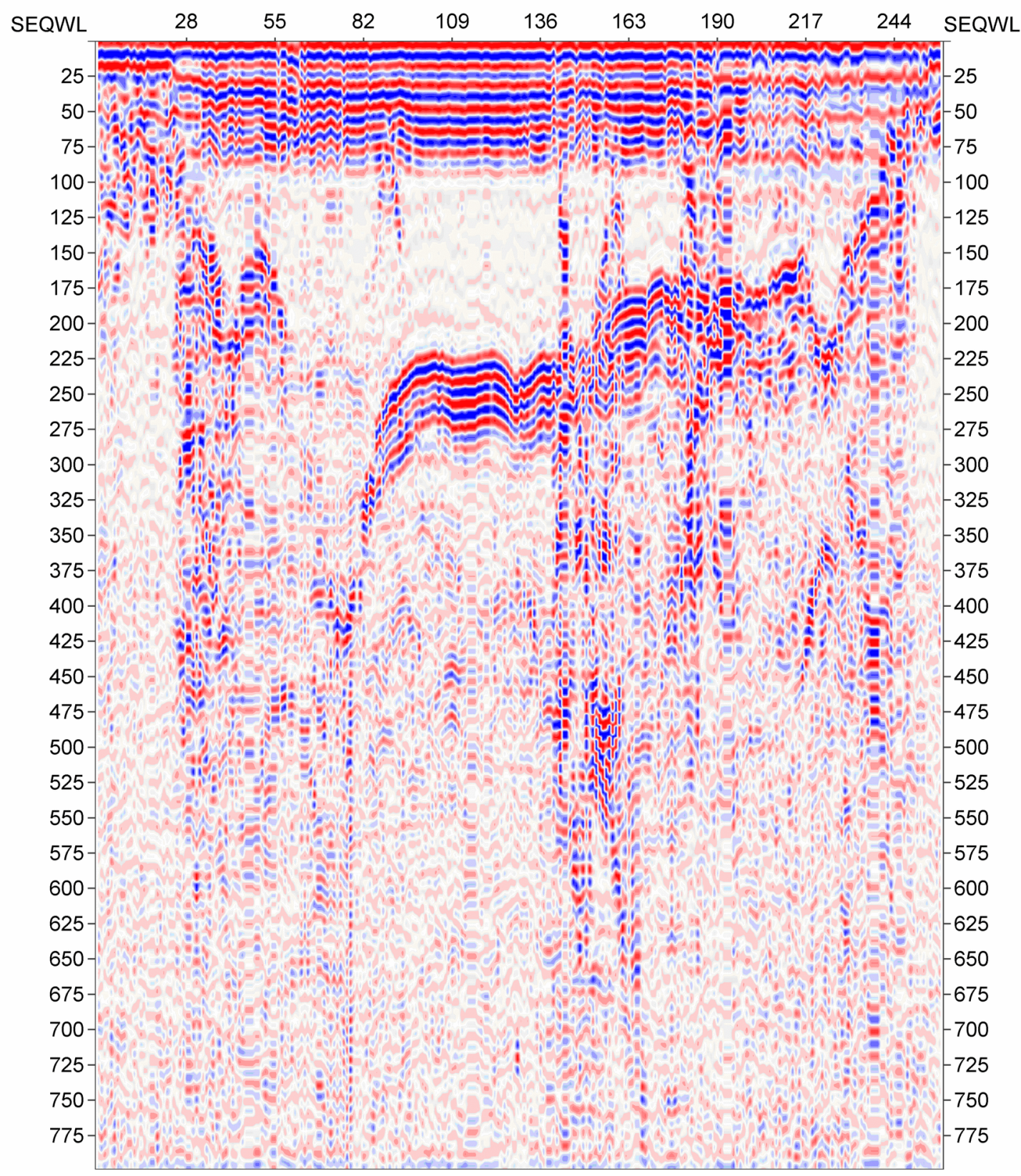




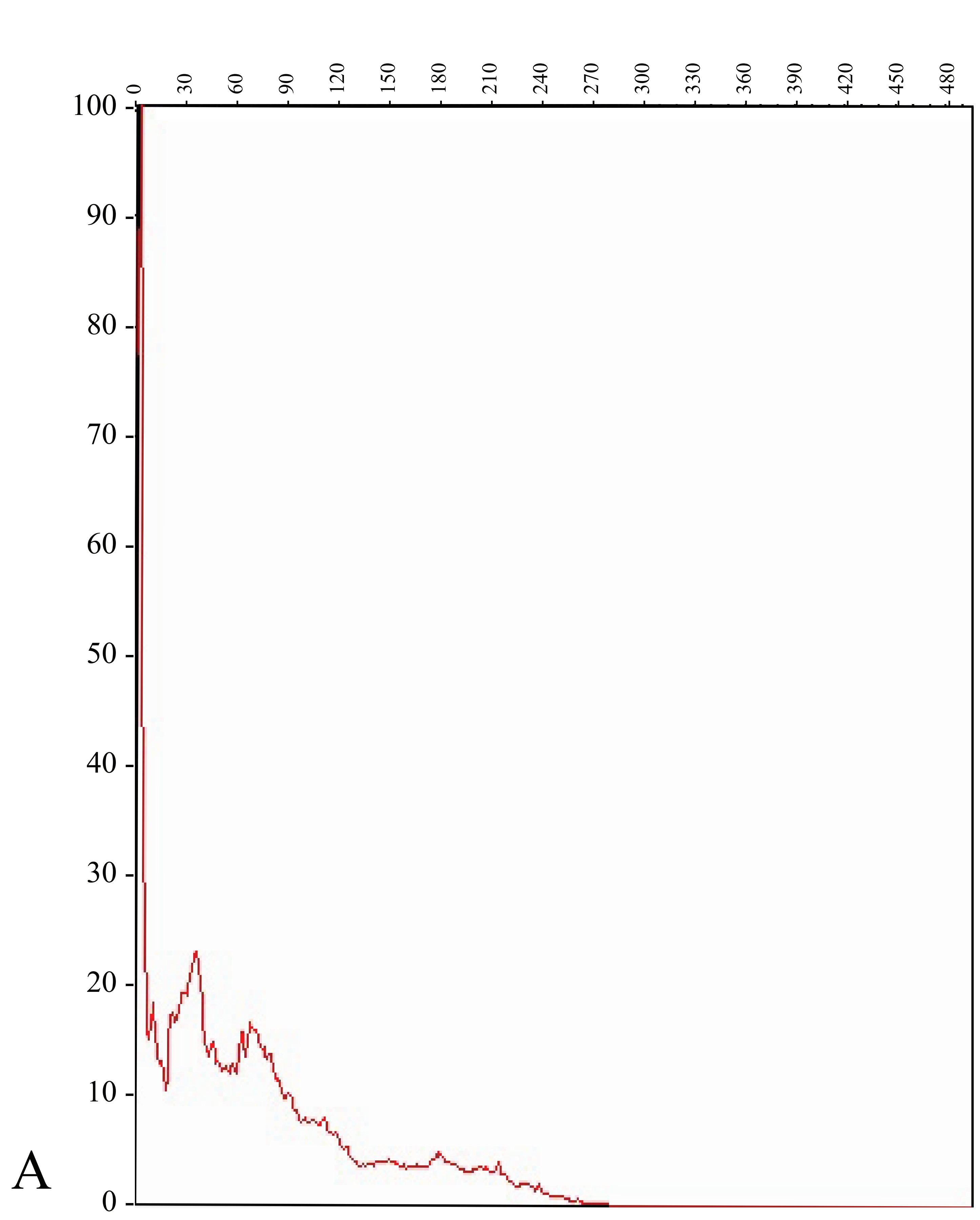

F 